I.V. Veshneva ${ }^{1}$, A.A. Bolshakov, R.A. Singatulin

\section{THE TECHNOLOGY OF A FEEDBACK CHANEL DEVELOPMENT FOR IDENTIFY THE STUDENT STATE IN THE LEARNING ERGATIC VIRTUAL REALITY SYSTEM}

\begin{abstract}
The application of the method of non-invasive diagnosis of the state of the student and mathematical processing of the data by status functions. To do this, we examine the technical methods of diagnostics and mathematical methods of data processing. Described used information measuring system.
\end{abstract}

Keywords: Status functions, virtual reality, ergatic system, photogrammetry, measuring information system

\section{Introduction}

The main prospect of a transformation occurring in education is the emergence, development and implementation of virtual reality training systems (VRTS) in the learning process (Veshneva,2015). Modern technologies allow to implement the most effective training - with using of the all senses (Shabrov). Virtual reality is a new artificial world designed by technological devices, transmitted to a person through his senses. The new concept of using a humanmachine interface for the create a threedimensional environment effect, in which the user interacts interactively with virtual objects, instead than with images of these objects (Thakral , Manhas and Kumar, 2010). Experience shows that the use of OS $\mathrm{BP}$ provides high learning motivation and success of learning by activating brain activity, $100 \%$ realistic images and the inclusion of all the senses. Innovations make it possible to move to a new qualitative level of information processing, modeling and designing experiments, creating complex machines and mechanisms, industrial facilities and processes. Highlight the main problems of development of VRTS. For the first, these are technical and software technologies for the creating VR. The next from the major problem is the development of systems for assessing the student's cognitive, motivational and psychological of the educable person. (Veshneva, 2014) (Bolshakov,Veshneva, Melnikov and Perov, 2014). This is the problem of developing mathematical models of the condition of the student person, the study of their psychological validity, the means of technical and software implementation of assessment systems (Singatulin and Gryshchenko 2010). Consideration of ways to design a feedback channel in ergatic VRTS devoted the present work.

\section{Main part}

\subsection{The technical possibilities for the estimate of the changes in the emotional state of a person}

Consider the technical possibilities of

\footnotetext{
${ }^{1}$ Corresponding author: I. V. Veshneva

Email: veshnevaiv@gmail.com
} 
implementing the monitoring of the psychophysical state of a person in contact with the learning environment. For the analyze different problems the are necessary evaluate the change in emotions. Emotions are a mechanism for directly assessing the level of well-being of an individual's interaction with the environment. They are either positive or negative, objects satisfy or do not satisfy needs. Elementary emotions have the simplest chemical and physical effects on the body. They are directly related to the endocrine-vegetative system and, if necessary, change the type of behavior. Emotions are the main motivating behavior forces. (Shingarov).

Traditional investigations of the behavior of individuals or groups of people to identify changes in the psychophysical state of the individual use the study of lexical and semantic deviations and changes in the motility of movements. (Kizhaev-Smyk, 1983).

Lexical-semantic deviations. By means of language, a person distinguishes, orders, and classifies objects and phenomena of reality (Abdullaeva, 2004). Semantic analysis of the text allows to identify the categories of the object, purpose and process to evaluate its pragmatic potential (Zlokazov, 2015). These methods can be widely used in social networks (Avsim).

Change in motility. Sign communication expresses unconscious connections and reactions. The gait, the position of the arms and legs, the position of the body, the movements of body parts, etc. can indicate a lot about both the emotional state of a person and his social position. At the same time, it can be quite difficult to separate the "true" movement, resulting from the relevance of feelings and moods, and the "targeted" movements reflecting volitional intentions. Researchers suggest a number of specific changes in motility characteristic of certain emotional states (Pease, 1992).

For an objective assessment of systemic changes in the organization of the brain, adequate quantitative assessment techniques are needed. Among such methods, it is worthwhile to single out electroencephalographic methods, vibro-image processing, and contactless scanning.

Electro-encephalogram (EEG) is a recording of the electrical activity of neurons of various brain structures, which is done on special paper using electrodes. Electrodes overlap on different parts of the head, and record the activity of a specific part of the brain. It can be argued that an electroencephalogram is a recording of the functional activity of the human brain.

In (Tsitrooshin, 1986), a method was developed for the study of the spatialtemporal relations of oscillations of brain biopotentials. It includes a multiparameter analysis of a multi-channel EEG with a graphical representation of the results as a set of EEG vectors in a three-dimensional factor space. When using it, the phenomenon of high orderliness of spatio-temporal relations of brain biopotential oscillations, characteristic of healthy children and adults at rest, was revealed (Shepovalnikov and Tsitroshin, 1999).

To analyze changes in brain activity in subjects during a deviant state modeled in hypnosis, the angular deviations of each of the EEG vectors from the position corresponding to the data in the state of calm wakefulness of the same subject are estimated.

Evaluating the magnitude of these radius vectors and the angles between them in successive standard eEG analysis periods of EEG (4 sec duration), it is possible to determine quantitatively and qualitatively the changes in the spatial structure of the biopotential field of the brain as a whole. In addition, cluster analysis was used to assess the degree of statistical connection between the parameters, reflecting the dynamics of the spatial structure of various combinations of interconnections of the bioelectric activity 


\section{Intermational Quality Conference}

QUA II IIY

of different parts of the cortex when performing test tasks.

The advantages of the method of assessing the structure of the biopotential field of the brain make it possible to analyze subtle functional restructuring of the systemic activity of the brain that occurs in various emotional states that may not be externally manifested by certain behavioral responses.

Vibration picture is a functional analogue of EEG. The frequency of the EEG signals is limited to the range of $0-30 \mathrm{~Hz}$. It is known that the mechanical movement of parts of the human body does not exceed $10 \mathrm{~Hz}$. This psychophysical phenomenon allows the use of standard webcams for high-quality recording of human vibro images. This conclusion allows us to expect a wide range of applications of methods for assessing the psychophysical state of a person using vibraimage technologies. It is important to note that there is a fundamental possibility of identifying a person in a crowd when several people are analyzed in a frame. In this case, the resolution of the object of study should not deteriorate. The most informative are the movements of the head and hands.

The increase in the number of terrorist acts in cities and traffic flows requires the creation of special measures to protect and monitor the state of persons posing a threat to public safety. One of the possible ways to ensure public safety is the installation of surveillance cameras and processing of filming with the use of vibrating image technologies. This technology records minor vibrations of a living object using standard hardware. The subtle vibration of the points of a person's body reflects the level of his emotional state, and also reveals instances of the desire to consciously control the state, such as controlling breathing, restraining irritation, or trying to hide emotional arousal. It is also possible to identify positive aspirations, such as interest in a particular phenomenon.

Contactless scanning. Non-invasive diagnostics using multispectral technologies is quite a promising area of the medical applications. Diagnostics is based on photogrammetric methods for measuring the position of the human body in various regions of the spectrum. The technology can be used in the diagnosis of motor disorders after a stroke and traumatic brain injury; in degenerative and hereditary diseases of the nervous system; as imaging techniques in cardiology; in diagnosing pain in the locomotor apparatus, etc. The technology has been developed in the information technology laboratory in humanitarian and natural science research of Saratov State University since 2002. The novelty of the proposed approach lies in the use of web cameras of multispectral wavebands and a special software algorithm. The diagnostic system binds objects or fragments of images received from webcams to the coordinate system by automatically identifying them with the corresponding fragments from the database. The orientation and scale of the original fragments can be arbitrary. To identify the fragments, the stereoidentification algorithm is used, allowing to compare images of different geometry. The sequence of procedures based on photogrammetric and multispectral technologies solves the problem of recognizing and distinguishing characteristic body contour shapes. The implementation of the proposed procedure was calculated during the diagnosis of a typical motor pattern (smooth movement with constant movement), non-optimal dynamic stereotype (appearance of additional compensatory synkinesis in the spine and extremities), atypical motor pattern (appearance of additional movements and distortion of the trajectory and speed) and some other cases. Measurement of motor deviations can occur in various places (at home, on the street), directly in the field of view of the multispectral system. At the output of the system, a three-dimensional description of the shape and dynamics of the object in a 
given format is formed. Output can be transmitted over the network. The data can also be visualized as a virtual threedimensional object with its own texture. The advantages of the method should be considered low cost, high measurement accuracy, ease of use and a high degree of automation, efficiency (diagnostics are performed in real time).

\subsection{Mathematical methods for creating the feedback channel of the ergatic systems}

The conditions of the physical medium of transmitting information about the state of the object initiate requirements for the development of appropriate software. At the same time, the theoretical stage of developing technical means for realizing the possibilities of determining changes in the emotional state of individuals or their groups requires the synthesis of a mathematical model of a continuous communication channel. The subsequent procedure for testing and debugging a technical device assumes the presence of a physical model or data of a full-scale experiment, constructed and processed on the basis of the original mathematical model. Possible models can be divided into indirect and direct description.

An indirect description requires an initial direct description, for example, a phenomenological one that directly connects the input and output signals of the communication channels. Currently, the most commonly used mathematical models of communication channels in the form of finite-dimensional conditional probability distributions (Levin and Schwartz, 1985), or certain moment functions (Klovsky, Kontorovich and Shirokov, 1984). In this case, it is necessary to use only the first two moments of the distributions due to the complexity of the apparatus used. The models are adequate only for Gaussian random processes (Wireless and Mobile
Networking Conference, 2013), which significantly narrows the range of applicability of the models being developed, since it significantly limits the memory in the channel.

Direct description is based on the use of differential (Levin and Schwartz, 1985) or integral equations (Shepovalnikov and Tsitroshin, 1999;. Kaiser and Zheng, 2010). Many researchers use a mathematical model of a communication channel in the form of some operator, for which decomposition bases for input and output signals are given in the form of harmonic functions (Golden, Dedieu, Jacobsen and Auerbach 2006). Direct description assumes consideration of the operator converting a set of input signals into output. In this case, the synthesized models rely on space, the coordinate functions of which are eigenfunctions of linear systems on an infinite interval of analysis, which ensures the universality of the models. An analysis of possible differential and integral operators is given in (Batenkov, 2010). The model of the communication channel is represented in the form of the operator:

$$
\mathrm{x}^{\prime}\left(\mathrm{t}^{\prime}, \mathrm{r}^{\prime}\right)=\mathrm{H}^{\prime}\{\mathrm{x}(\mathrm{t}, \mathrm{r})\}+\mathrm{n}\left(\mathrm{t}^{\prime}, \mathrm{r}^{\prime}\right)
$$

where $H^{\prime}$ is the operator display the useful component of the received mixture, depending on the properties of the transmission medium, as well as the characteristics of the input and output devices (matching); $n\left(t^{\prime}, r^{\prime}\right)$ is the field of additive noise, which sets both external noise and internal noise; $t, r$ - temporal and spatial coordinates, respectively.

Operator $H^{\prime}$ is a system characteristic and reproduces the characteristics of the signal observed at the output. The description of the feedback channel comes down to the development of its system characteristic. The description can be used for nonlinear and for linear systems. When describing nonlinear systems, the development of partial differential equations is required. The system characteristic itself is the coefficients of this 


\section{Intermational Quality Conference}

differential equation. The explicit form of the operator $\mathrm{H}^{\prime}$ is determined by many factors: the type of equation, the form of space-time signals at the input $x(t, r)$, and the initial conditions. However, in the general case, analytical solutions for an arbitrary type of equations do not exist.

The representation of a system characteristic in the form of an integral operator represents the possibility of approximating a continuous functional with a given accuracy by a Volterra functional series (Tikhonov and Kharisov, 1991). Differential equations allow to describe the relations between input and output signals on the basis of coefficients, and Volterra series allow taking weight functions into integral transformations into account.

Both types of representation of system characteristics are greatly simplified if the simulation is carried out on the basis of linear operators. For differential equations, the requirement of realness and independence from the signals of the coefficients of the differential equation is introduced, which leads it to a linear form. The Volterra series is significantly reduced, degenerating into a single integral transform (convolution integral, or Cauchy integral), the weight function of which is the impulse response.

\subsection{Description of the experiment of using contactless scanning of the person emotional state in the educational process}

We will develop a model of the feedback channel OS RV. The mathematical model of assessing the condition of the student is based on the method of complex status functions (SF) (Veshneva, 2014). The equipment of the information measuring system (IMS) includes: a base unit containing a video system; structured (infrared) illumination system; hardwaresoftware unit consisting of a PC and the corresponding software; interface for working with network data; physiological parameters analyzer.

The base unit uses a system of two or more video cameras for technical vision and a special source of structured lighting. For each current position of the object of research, IIS provides a calculation of the spatial coordinates of the illuminated crosssectional points of the object located in the working area of the measuring complex. By moving the points of the illumination on the object, a sequential scanning of the entire required surface of the object and the calculation of the sequence of coordinates of the object's sections, according to which the shape, size, dynamics and state of the object are evaluated, are performed. At the output of the system, a three-dimensional description of the object is formed in a given format, or a set of measurements of a given type.

The output is transmitted over the network. The base unit for the optical-geometric analysis is formed on the basis of two CCDmatrices. The spectral range affects the visible range, as well as infrared (IR), in the range of 0,8-2,2 microns. The effective range of the used IIS is about $50 \mathrm{~m}$. The graphic station uses an IBM-compatible computer with the following characteristics: Intel Core i5-2405S processor with a frequency of 1.33 $\mathrm{GHz}, 8 \mathrm{~GB}$ RAM, integrated video adapter with video memory capacity of $2048 \mathrm{MB}$, $750 \mathrm{~Gb}$ hard drive.

The software package includes: an operating system with a full ALT Linux software package (with stereo support), an Avango virtual reality system, a PHOTOMOD digital photogrammetric system from Racurs, and its own software. The features of the developed educational technology provide the opportunity to conduct individual and group classes in real time and move on to methods of simultaneously studying a specific training topic using the "immersion method". The system actively uses a noninvasive student feedback method based on multispectral analysis and a neural network 
QUA IIIIY

DIFSIFAIDCI

expert system. The analyzing device takes into account the features of the facial expression, head position, dynamics of the movement of eyes, lips, hands and intensity (saturation) of the body color (face) of each student. Measurement results are processed both statistically using discriminatory analysis and on the basis of new algorithms for optical-geometric analysis.
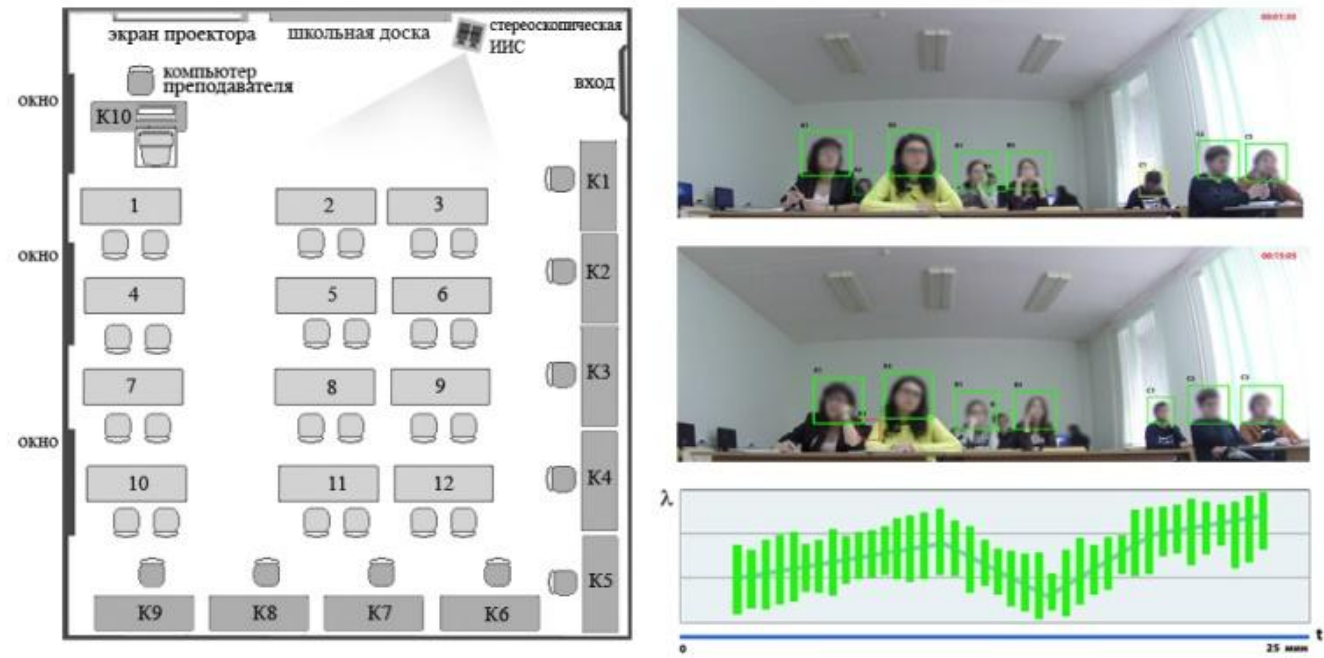

Figure1. The work of frontal IMS in the classroom and its layout.
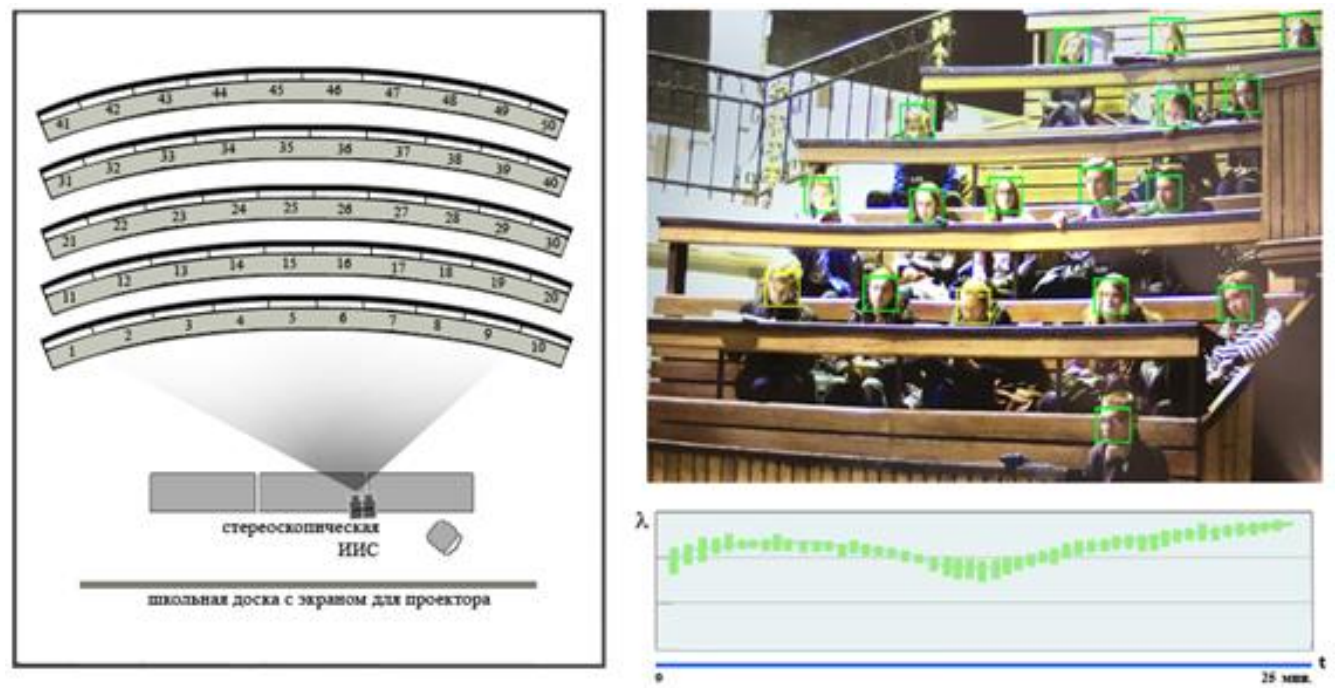

Figure 2. The work of frontal IMS in a large audience and its layout.

The training audience, as a rule, being a methodical center for ensuring the computer competence of students, teachers and employees, is completed for this purpose with appropriate office equipment, expendable, methodical and educational materials. 
Organization of premises, workplaces of students, teachers, visual materials and auxiliary equipment - should be regulated by the existing regulatory documents. At the same time, in-depth training of students and the construction of the educational process in an ICT-rich educational environment (using existing learning environments and / or general-purpose development tools, various standards and approaches) leads to a significant violation of regulatory instructions.

During training sessions, not only elementary sanitary and hygienic norms, ergonomic, psychophysical parameters, but also other safety rules related to the placement and operation of various equipment are not observed. This significantly affects the input measured parameters of IIS, the quality of student learning of the material, especially in situations with long classes (fig. 1, 2).

The negative factors can be attributed to a significant reduction in the effectiveness of used teaching materials and instrumentation equipment, due to the irrational consideration of the formed realities when using modern information communication technology.

A solution for more efficient measurement using IMS can be the creation of duplicate systems that can be placed not only frontally, but also used as an additional rear system. Such an arrangement will allow synchronizing IMS, not obscuring, from different angles, which will undoubtedly have a positive effect on the accuracy of measurements (fig. 3).

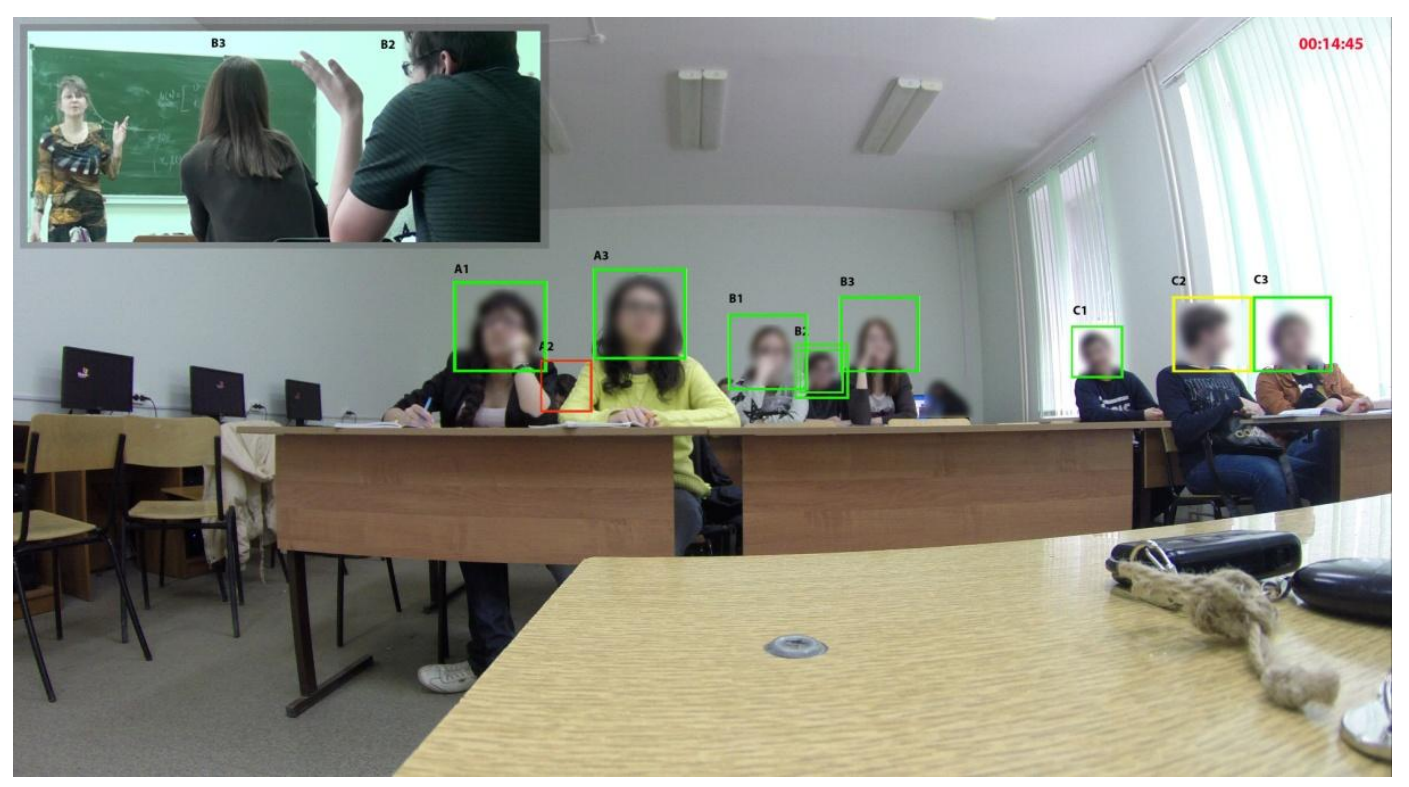

Figure 3. Work frontal IMS in the audience. In the upper left frame, the operation of the rear IIS for synchronization with the front camera.

The efficiency of measurements will be much higher if we foresee a fan, stepwise placement of the objects of study in the field of IMS. In any case, the placement of IMS in a specific classroom requires preliminary evaluation work. 
The advantages of the proposed technology in relation to other educational systems (VR, AR-systems, integrated distance learning systems, computer simulators, simulators, etc.) are based on: the use of low-cost digital (non-metric) stereo cameras included in the IMS basic structure; obtaining real-time information in real time, both directly from the camera (using the IP family of the protocol), and through a special video server for wide access, for an expanded audience; use of proprietary software for accelerated processing of spatial data.

The technology used is based on multispectral and photogrammetric methods, and is characterized by high speed and measurement accuracy, ease of use and a high degree of automation. It can be used without fundamental transformations in conditions of strong "noisiness" of the audience, independence from network data and changes in the composition of measurement objects in real time.

Changing the functional and qualitative characteristics of the educational process, classrooms and workplaces with the help of advanced ICT developments, will make it possible in the near future to make fundamental, positive changes in the teaching of general and higher education in the structure of the new integrated education.

\section{References}

Veshneva I.V., Singatulin R.A. Development of information measuring systems for distance learning complex with feedback based on photogrammetric methods and status functions. Part 2 // Bulletin of Saratov State Technical University. - 2015. - T. 3. № 1 (80) - C. 125132.

Shabrov N.N. Software and hardware complexes of virtual reality of predictive modeling in scientific and engineering research [Electronic resource] // Supercomputer consortium of universities of Russia. Access mode: hpc-russia.ru/23.pdf. The title from the screen.

Thakral S., Manhas P., Kumar C. Virtual Reality and M-Learning // International Journal of Electronic Engineering Research. 2010. Vol. 2, No. 5. P. 659-661.

Veshneva I.V. The use of the Karunen-Loeve modes in the intellectual system of monitoring the process of formation of professional competencies // Bulletin of Kazan Technological University. T. 17 , № 17. 2014. C. 195-202.

New methods of mathematical modeling of the dynamics and management of the formation of competences in the process of learning in high school: monograph / A.A. Bolshakov, I.V. Veshneva, L.A. Melnikov, L.G. Perov. M .: Hotline - Telecom, 2014. 250 p.

Singatulin R.A., Gryshchenko E.A. The use of multispectral diagnostic systems in a virtual educational environment // Information technologies in providing a new quality of higher education. Sat scientific Art. M .: ICPPC., Vol. 2. 2010. C. 246-249.

Shingarov G.K. Emotions and feelings as a form of reflection of reality.

. Kizhaev-Smyk L. Psychology of stress. M.Nauka, 1983.

- Abdullaeva M.M. Professional Identity of Personality: Psychosemantic Approach // Psychological Journal. 2004. V. 25. No. 2. P. 86-95.

Zlokazov K.V. Content analysis of destructive texts // Political linguistics. 2015. 1 (51). Pp. 244-251

. Avsim.su: Aggression in social networks [Electronic resource]. - 2010. - Access mode: http: 
www. avs im.su / forum / blo g / 84 / entry-122-agressiva-v-socialnih-setvah /.

Pease A. Body Language. How to read the thoughts of others by gestures. Nizhny Novgorod: IQ, 1992

Tsitrooshin M.N. Analysis of the statistical relationship of oscillations of brain biopotentials in a three-dimensional factor space // Avtometriya. 1986. № 6. S. 89.

Shepovalnikov A.N., Tsitroshin M.N. Evolutionary aspects of the formation of the integrative activity of the human brain // Russian physiological journal. THEM. Sechenov. 1999. T. 85. № 9-10. Pp. 1187.

Levin, B.R., Schwartz, V. Probabilistic Models and Methods in Communication and Control Systems. Moscow: Radio and Communications, 1985. 312 p.

. Klovsky D.D., Kontorovich V.Ya., Shirokov S.M. Models of continuous communication channels based on stochastic differential // Ed. D.D. Klovsky. M .: Radio and communication, 1984. $247 \mathrm{p}$.

Modeling using Continuous Time Markov Chains // In the 6th Joint IFIP Wireless and Mobile Networking Conference (WMNC) 2013. Dubai, United Arab Emirates (UAE), April 2013.

Kaiser T., Zheng F. Ultra wideband systems with MIMO. Chichester: John Wiley \& Sons Ltd, 2010. 254 p.

Fundamentals of DSL technology // edited by Golden P., Dedieu H., Jacobsen K. NY: Auerbach Publications, 2006. 454 p.

Batenkov K. A. Continuous channel modeling in shape of some space transformation operators // Труды СПИИРАН. 2014. 1(32). ISSN 2078-9181 (печ.), ISSN 2078-9599 URL: www.proceedings.spiiras.nw.ru (In Russian)

Tikhonov V.I., Kharisov V.N. Statistical analysis and synthesis of radio engineering devices and systems // studies. manual for universities. M .: Radio I svyaz, 1991. 608 p. (In Russian)

Veshneva I.V.1, Travina T.L. Description of professional competence development using complex fields of status function: a new approach to the problem // Bulletin of Saratov State Technical University. - 2012. - T. 3. № 1(67). - C. 175-182. (In Russian)

Model of formation of the feedback channel within ergatic systems for monitoring of quality of processes of formation of personnel competences / I. Veshneva, R. Singatulin, A. Bolshakov, T. Chistyakova, Melnikov L. // International Journal for Quality Research, 2015. vol. 9, num. 3. p. 495-512.

\section{I.V.Veshneva}

State National Research University,

Saratov,

Russia

veshnevaiv@gmail.com
A.A. Bolshakov

Peter thre Great St. Petersburg Polytechnic

University

St. Petersburg,

Russia

aabolshakov57@gmail.com

\section{R.A. Singatulin}

State National Research

University,

Saratov,

Russia

labsgu@mail.ru 


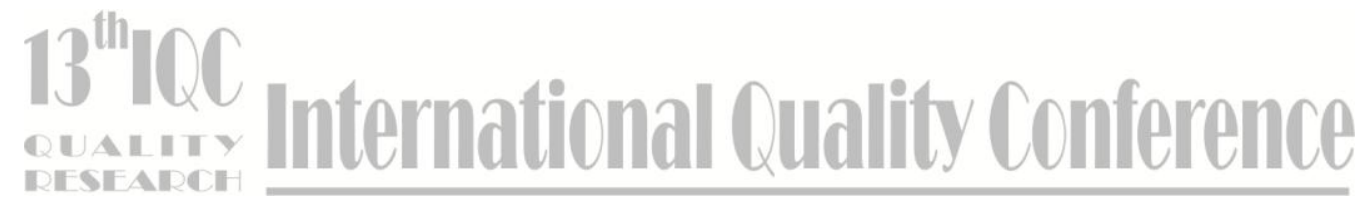

